\title{
Review of 113 Anaesthetics for Patients Undergoing Cytoreductive Surgery and Hyperthermic Intraperitoneal Chemotherapy (HIPEC)
}

\author{
Sze Ying Thong, Shulyn Claramae Chia, Oriana Ng, Hwei Ching Grace Tan, Ee Teng \\ Ong, Khee Chee Soo, Ching Ching Melissa Teo
}

\section{Background}

Cytoreductive surgery (CRS) and hyperthermic intraperitoneal chemotherapy (HIPEC) are the treatment of choice for selected patients with peritoneal carcinomatosis, which was previously a lethal condition with dismal survival rate. CRS aims to remove macroscopic disease while HIPEC works synergistically by removing microscopic tumour load at 42 $43 \mathrm{C}$.

\section{Methods}

We reviewed all CRS and HIPEC procedures performed in Singapore General Hospital/ National Cancer Centre from January 1997 to December 2012, with a focus on perioperative events and anaesthetic implications.

\section{Results}

In total, 111 patients underwent 113 procedures. Mean age of patients was $51.7 \pm$ 11.6. Postoperatively, $78.8 \%$ of patients required intensive care. Median length of ICU stay was 2 days. $75.2 \%$ (85) required post-operative ventilator support, of which $69.4 \%$ (59) and $12.9 \%$ (11) were extubated on the first and second postoperative days. Patients with lower PCI scores (7 vs 17) were more likely to be extubated immediately post-operation $(\mathrm{P}<0.05)$. Patients with a longer HIPEC duration (63 vs 58 minutes) were more likely to have post-operative coagulopathy. Median duration to discharge from hospital was 14 days. Patients who required longer hospitalization ( $>14$ days) had longer durations of surgery (603 vs 498 minutes) than those who had shorter hospitalization stay $(\mathrm{P}<0.05)$.

\section{Table 1. Demographics of patients undergoing CRS and HIPEC (n=113)}

\begin{tabular}{ll}
\hline Variable & Number (\%) \\
\hline ASA status & \\
I & $62(54.9 \%)$ \\
II & $45(39.8 \%)$ \\
III & $6(5.3 \%)$ \\
Primary Tumour & \\
Ovarian & $43(38.1 \%)$ \\
Colorectal & $35(31.0 \%)$ \\
Appendix Mucinous Adenocarcinoma & $12(10.6 \%)$ \\
Pseudomyxoma peritonei & $11(9.7 \%)$ \\
Mesothelioma & $8(7.1 \%)$ \\
Primary peritoneal carcinoma & $3(2.7 \%)$ \\
Gastric carcinoma & $1(0.9 \%)$ \\
Gender & \\
Male & $18(15.9 \%)$ \\
Female & $95(84.1 \%)$ \\
Ischaemic heart disease & \\
Yes & $3(2.7 \%)$ \\
No & $110(97.3 \%)$
\end{tabular}

\section{Conclusion}

The combination of CRS and HIPEC is a major complex procedure with significant morbidity and many anaesthetic considerations. Many patients who present for surgery are relatively young and without significant comorbidity, but the extensive procedure will result in multisystem derangements that include the cardiorespiratory system, fluid and acid base balance as well as body temperature. Our study has highlighted some of the perioperative concerns associated with CRS and HIPEC. The development of an optimal anaesthetic technique in conjunction with evidence-based medicine may improve outcomes in this group of patients.

\section{References}

1. Teo M. Peritoneal-based malignancies and their treatment. Ann Acad Med Singapore 2010; 39: 54-7

2. Bryant J. Systematic review of the Sugarbaker procedure for pseudomyxoma perionei. Br J. Surg 2005; 92: 153-8

\section{National Cancer

\section{Singapore} General Hospital
Table 2. Intraoperative variables
Intraoperative Variable

Surgery duration

Peritoneal Cancer Index Score

Estimated Blood loss (ml)

Hourly Urine output (ml/kg/hr)

Crystalloid administered (ml)

Total IV and blood products administered (ml)

Intravenous morphine administered (mg/kg)

Blood product/colloid administered

Blood (ml) $(\mathrm{n}=87 ; 77.0 \%)$

FFP $(\mathrm{ml})(\mathrm{n}=21 ; 18.6 \%)$

Platelet $(\mathrm{ml})(\mathrm{n}=7 ; 6.2 \%)$

Cryoprecipitate $(\mathrm{ml})(\mathrm{n}=1 ; 0.9 \%)$

Total colloids $(\mathrm{ml})(\mathrm{n}=88 ; 77.9 \%)$

Temperature

At the start

Minimum Intraop Temperature

Maximum Intraop Temperature

Temperature at end of surgery

Temperature difference (end of surgery to start)

Peak airway pressure $(\mathrm{cmH} 2 \mathrm{O})$

$$
\begin{aligned}
& \text { Minimum } \\
& \text { Maximum } \\
& \text { Change }
\end{aligned}
$$

Final $\mathrm{pH}$

\begin{tabular}{|c|c|}
\hline Variable & Number $(\%)$ \\
\hline $\begin{array}{l}\text { Intraoperative Hourly Urine output } \\
\quad<0.5 \mathrm{ml} / \mathrm{kg} / \text { hour } \\
\quad>0.5 \mathrm{ml} / \mathrm{kg} / \text { hour }\end{array}$ & $\begin{array}{l}3(3.3 \%) \\
88(96.7 \%)\end{array}$ \\
\hline $\begin{array}{l}\text { Temperature at end of surgery } \\
\quad<36.0 \\
36.0-37.4 \\
>37.4\end{array}$ & $\begin{array}{l}12(12.6 \%) \\
62(65.3 \%) \\
21(22.1 \%)\end{array}$ \\
\hline $\begin{array}{l}\text { Postoperative discharge disposition } \\
\text { Intensive care unit } \\
\text { Intermediate care unit } \\
\text { High dependency area }\end{array}$ & $\begin{array}{l}89(78.8 \%) \\
18(15.9 \%) \\
5(4.4 \%)\end{array}$ \\
\hline $\begin{array}{l}\text { Postoperative extubation } \\
\text { Immediately post surgery } \\
\text { First postoperative day } \\
\text { Second postoperative day } \\
\geq \text { Third postoperative day }\end{array}$ & $\begin{array}{l}28(24.8 \%) \\
59(52.2 \%) \\
11(9.7 \%) \\
15(13.3 \%)\end{array}$ \\
\hline $\begin{array}{l}\text { Death during same admission } \\
\text { Yes } \\
\text { No }\end{array}$ & $\begin{array}{l}2(1.8 \%) \\
111(98.2 \%)\end{array}$ \\
\hline $\begin{array}{l}\text { Number of chest tubes inserted } \\
0 \\
1 \\
2\end{array}$ & $\begin{array}{l}37(32.7 \%) \\
23(20.4 \%) \\
53(46.9 \%)\end{array}$ \\
\hline $\begin{array}{l}\text { Postoperative coagulopathy } \\
\text { Elevated prothrombin time } \\
\text { Elevated activated prothrombin time }\end{array}$ & $\begin{array}{l}79(79.0 \%) \\
44(44.0 \%)\end{array}$ \\
\hline Postoperative renal impairment & $6(5.6 \%)$ \\
\hline
\end{tabular}

Final Base excess

Table 3. Other perioperative events $(n=113)$

\begin{tabular}{l}
$\begin{array}{l}\text { Mean } \pm \text { Standard } \\
\text { deviation }\end{array}$ \\
9 Hr $10 \mathrm{~min} \pm 2 \mathrm{hr} 56 \mathrm{~min}$ \\
$14.3 \pm 8.9$ \\
$1481 \pm 1064$ \\
$1.6 \pm 1.0$ \\
$5852 \pm 2912$ \\
$8498 \pm 3941$ \\
\\
$0.4 \pm 0.2$ \\
$1089 \pm 722$ \\
$604 \pm 243$ \\
$476 \pm 279$ \\
150 \\
$1790 \pm 1309$ \\
$35.6 \pm 0.6$ \\
$35.0 \pm 0.7$ \\
$37.4 \pm 0.8$ \\
$36.8 \pm 0.9$ \\
$1.2 \pm 1.0$ \\
\\
$17.4 \pm 2.8$ \\
$25.8 \pm 5.1$ \\
$8.4 \pm 4.4$ \\
$7.33 \pm 0.07$ \\
$-5.33 \pm 2.89$ \\
\hline
\end{tabular}

